\title{
First detection of the growing humps at the rapidly rising stage of dwarf novae AL Com and WZ Sge
}

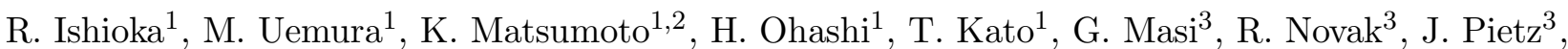
B. Martin ${ }^{3}$, D. Starkey ${ }^{3}$, S. Kiyota ${ }^{3}$, A. Oksanen ${ }^{3}$, M. Moilanen ${ }^{3}$, L. Cook ${ }^{3}$, L. Kral $^{3}$, T. Hynek ${ }^{3}$, M. Kolasa ${ }^{3}$, T. Vanmunster ${ }^{3}$, M. Richmond ${ }^{3}$, J. Kern ${ }^{3}$, S. Davis ${ }^{3}$, D. Crabtree ${ }^{3}$, K. Beaulieu ${ }^{3}$, T. Davis ${ }^{3}$, M. Aggleton ${ }^{3}$, K. Gazeas ${ }^{3}$, P. Niarchos ${ }^{3}$, A. Yushchenko ${ }^{3}$, F. Mallia ${ }^{3}$, M. Fiaschi ${ }^{3}$, G. A. Good ${ }^{3}$, D. Boyd $^{3}$, Y. Sano ${ }^{3}$, K. Morikawa ${ }^{3}$, M. Moriyama ${ }^{3}$, R. Mennickent ${ }^{3}$, J. Arenas ${ }^{3}$, T. Ohshima ${ }^{3}$, and T. Watanabe ${ }^{3}$

1 Department of Astronomy, Faculty of Science, Kyoto University, Kyoto 606-8502, Japan

2 Graduate School of Natural Science and Technology, Okayama University, Okayama 700-8530, Japan

3 VSNET Collaboration Team

Received 29 October 2001 / Accepted 20 November 2001

\begin{abstract}
We report on time-series photometric observations in the earliest stages of superoutbursts of the extreme dwarf novae, AL Com and WZ Sge, which started on 2001 May after the 6 years quiescence and on 2001 July after the 23 years quiescence, respectively. We detected the growth of "early superhumps" during the each rising stage. Our observations reject the mass transfer instability for the trigger of the superoutburst of WZ Sge stars, and show the existence of some relations between the "early superhumps" and the spiral structure, which gives a hint of the origin of the "early superhumps."
\end{abstract}

Key words. stars: cataclysmic variables - stars: dwarf novae - stars: individual (WZ Sge)

\section{Introduction}

Dwarf novae are cataclysmic binaries containing a Roche lobe-filling dwarf star and a white dwarf with an accretion disk (for a review, see Warner 1995). SU UMa-type dwarf novae (SU UMa stars) are a subclass of dwarf novae, which are characterized by two types of outbursts - short "normal" outbursts and long "superoutbursts". During superoutbursts, periodic modulations called "superhumps" are observed, whose period is slightly longer than the orbital period. The best accepted mechanism for SU UMa stars is a combination of thermal and tidal instabilities in the accretion disk (DI model; Osaki 1989), and the superhump phenomenon is well explained by the tidally distorted accretion disk (Ichikawa et al. 1993).

WZ Sge-type dwarf novae (originally proposed by Bailey 1979, see also Kato et al. 2001) form an extreme subgroup of SU UMa stars. The well-established members are only 5 stars, HV Vir, AL Com, EG Cnc, RZ Leo, and WZ Sge itself. They also show superoutbursts and superhumps, but have many peculiar respects different from usual SU UMa stars. The outburst frequency of WZ Sge stars is very low and the interval of two successive superoutbursts (supercycle) is years to decades (usually

Send offprint requests to: R. Ishioka,

e-mail: ishioka@kusastro.kyoto-u.ac.jp months), the outburst amplitude is more than 6 mag (usually $2-5 \mathrm{mag}$ ), the outburst duration is months (usually two weeks), and no or few normal outburst occurs in one supercycle (usually several ones).

However, these characteristics are relative ones, and there has been a suggestion that WZ Sge stars and SU UMa stars comprise a continuous entity, rather than a separate class (Osaki 1995). The only diagnostic characteristics is the existence of "early superhumps" superhumps are periodic modulations with a period identical to the orbital period and often with a complicated profile, which is observed in the earliest stage of the superoutburst of the WZ Sge stars. The early superhumps are observed only in the WZ Sge stars, and never in the other SU UMa stars. Thus the understanding of early superhumps is very important in the understanding of WZ Sge stars as a distinct group, but the origin is still an open question.

1 The same feature is also referred to as orbital superhumps (Kato et al. 1996) or outburst orbital hump (Patterson et al. 1998). We use the term in this paper "early superhumps" because the period of this feature was shown to be slightly, but significantly different from the orbital period (Sect. 2), qualifying the superhump nature of the signal, and because we consider that the origin of the feature is a tidal resonance, rather than an enhanced hot spot (Sect. 3). 
Many authors have tried to explain the origin of the peculiarity of the WZ Sge stars. Osaki (1995) showed that if the very low mass transfer rate and the extremely low viscosity parameter, $\alpha$, of the quiescent disk is assumed, the long cycle length and the large scale outburst can be explained in the disk instability model. Another explanation is a mass transfer burst model, which explains the outburst by the sudden increase of the mass transfer rate from the secondary (Patterson et al. 1981).

The distinction between the two models is evidenced by the observations of the outburst onset. However, the difficulty of the observational study of WZ Sge stars is in their long supercycle length (e.g. 32.5 years of WZ Sge), as well as, the shortness of the rising stage of the outburst of dwarf novae. During the last outburst of WZ Sge in 1978, unfavorable seasonal conditions made detailed and continuous photometric observations difficult. Two superoutbursts of AL Com in 1995 and EG Cnc in 1997 were rather well observed (Kato et al. 1996; Nogami et al. 1997; Matsumoto et al. 1998, and references therein). However, the rising stages of the outbursts were not covered by the observations in both outbursts, too. Many theoretical studies have been done based on the relatively fragmentary data of WZ Sge during previous outbursts and the characteristics of the other members of the WZ Sge stars, but more detailed data of WZ Sge stars during outbursts are necessary to test the theories. So the further outbursts of WZ Sge stars, and the precise observations, especially the observations of the earliest stage, have been strongly desired for a long time.

Here, we report on the first detection of the growth of periodic modulations in the earliest stage of the long-awaited two superoutbursts of AL Com and WZ Sge, which were discovered by S. Kerr on 2001 May 18.51 at $m_{\mathrm{vis}}=13.4$ and by T. Ohshima on 2001 July 23.565 UT at $m_{\text {vis }}=9.7$, respectively. For both outbursts, receiving the reports of the outbursts, we immediately started extensive international campaign of time-series photometric observations through the Variable Star NETwork (VSNET, http://www.kusastro.kyoto-u.ac.jp/vsnet/). Both campaigns succeeded in detecting the rising stages of the outbursts and revealed that periodic modulations (early superhumps) grew after the outburst onset. In this letter, we focus our interest on the earliest stages of the outbursts. The details of the observations of WZ Sge and $\mathrm{AL}$ Com will be issued in forthcoming papers.

\section{Observations and results}

The CCD observations reported here were performed by the international VSNET collaboration team using 5.5$80 \mathrm{~cm}$ telescopes with time resolutions of $0.5-100 \mathrm{~s}$.

The magnitudes of AL Com and WZ Sge were measured using several comparison stars (for WZ Sge, see Henden \& Landolt 2001), and adjusted to a common scale close to the $R_{\mathrm{c}}$ system by correcting for the systematic difference between observers. Heliocentric corrections to the observed times were applied before the following analyses.
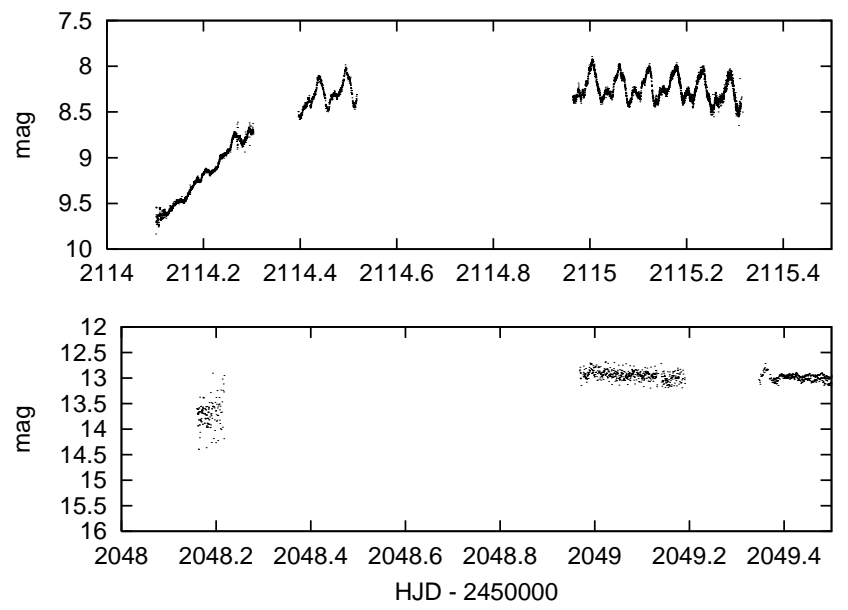

Fig. 1. Light curves of the earliest stage of the 2001 superoutbursts in AL Com (lower panel) and WZ Sge (upper panel). In the case of AL Com, no clear hump was observed in the rising stage, although a hint of hump appeared. In the case of WZ Sge, the growth of humps was clearly detected during the rising stage.

Figure 1 shows the light curves by the earliest observations of the two outbursts. Our observations detected the rapid brightenings with a rate of more than $3 \mathrm{mag} \mathrm{d}^{-1}$ for AL Com and more than 5 mag d $^{-1}$ for WZ Sge. In both outbursts, no clear modulation was observed just after the outburst onset, but the full amplitude modulations grew up before the outburst maxima. The light curve of WZ Sge clearly shows the growth of periodic modulations superimposed on the rising trend. The growing time scale of the periodic modulations was only one day.

Figure 2 shows the whole light curves of the outbursts. Since the presence of two types of superhumps in AL Com during its 1995 superoutburst was already described in the literature (Kato et al. 1996), we mainly focus on WZ Sge in this letter, especially on the clarification of two types of humps.

As shown in the upper panel of Fig. 2, the main outburst continued for 23 days. The declining rate was more than $0.2 \mathrm{mag} \mathrm{d}^{-1}$ at first, however, finally settled into $0.13 \mathrm{mag} \mathrm{d}^{-1}$ which is a typical value for the SU UMa stars.

We measured the peak timings of the humps during the main outburst and performed a period analysis. The cycle counts start by $E=0$ at HJD 2452115.0330. There is no uncertainty concerning our cycle count because of quite dense observations. The typical error of the timing is $0.001 \mathrm{~d}$. In the case that the hump is double-peaked, the timings of both peaks were measured for one cycle count. Figure 3 shows the $\mathrm{O}-\mathrm{C}$ diagram calculated for the orbital period of $0.05668784707 \mathrm{~d}$ (Skidmore 1998).

During the period of July 23-August $3(0 \leq E \leq$ 206 ), the major peak timing of recurring humps with a stable period is best fitted by the following equation: Peak(HJD) $=0.056656(2) E+2452115.0000(2)$. The period of $0.056656(2) \mathrm{d}$ is $0.05 \%$ shorter than the orbital period of $0.05668784707 \mathrm{~d}$ (Skidmore 1998), but the two 


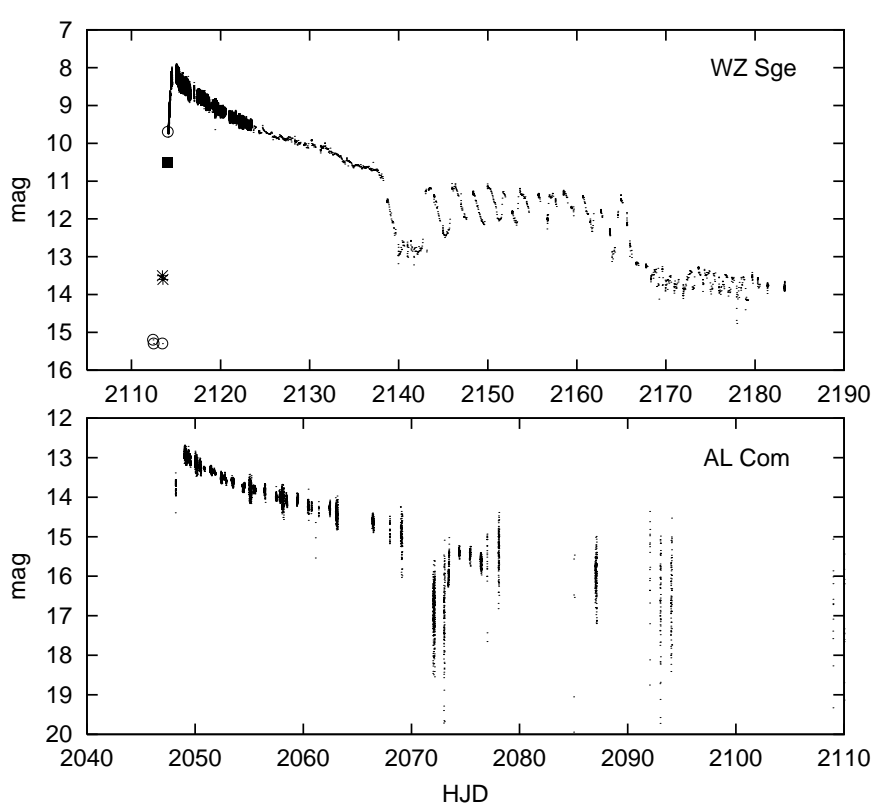

Fig. 2. General light curves of the 2001 superoutbursts in AL Com (lower panel) and WZ Sge (upper panel). Small dots represent our CCD observations, filled square represents photographic observation, open circles represent visual observations, and crosses represent negative observations.

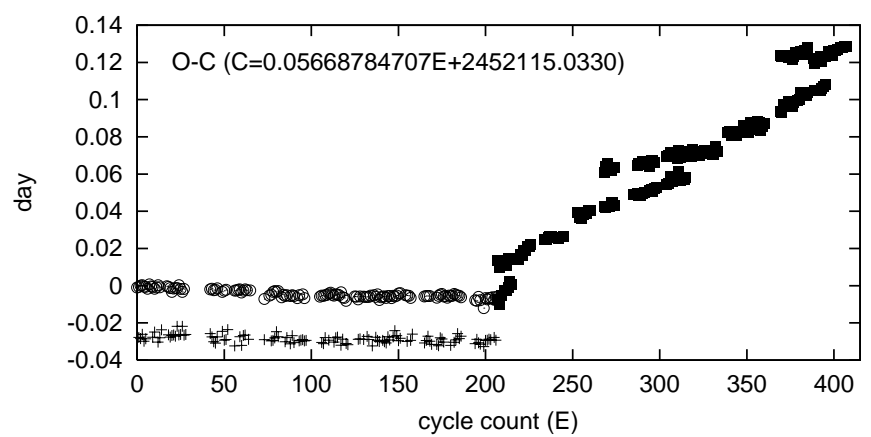

Fig. 3. The O-C diagram of the hump-maxima timings, open circles represent major peaks of early superhumps, cross represent minor peaks of early superhumps, and filled squares represent peaks of superhumps.

periods are very close to each other. On August 4, a different type of humps with a longer period appeared. During the period of August 4-16 (206 $\leq E \leq 407)$, the peak timing is fitted by another equation: Peak(HJD) $=$ $0.05726(1) E+2452114.890(4)$. The period of 0.05726(1) d is $1.01 \%$ longer than the orbital period. These two periods were also confirmed by a period analysis using PDM method.

Figure 4 shows the averaged light curves of two types of humps. The former type of humps with a period of $0.056656(2) \mathrm{d}$, almost identical to the orbital period, is "early superhumps." The latter type of humps with a period of $0.05726(1) \mathrm{d}, 1.0 \%$ longer than the orbital period, is "superhumps."

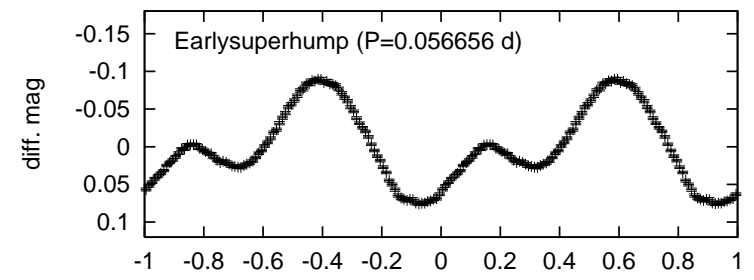

Fig. 4. Averaged light curves of early superhumps and superhumps of WZ Sge. Upper panel shows an averaged light curve of early superhumps folded by a period of $0.056656 \mathrm{~d}$. Lower panel shows an averaged light curve of superhumps folded by a period of $0.05726 \mathrm{~d}$.

Although there had been a suggestion of two types of periodic modulation during the 1978 outburst (Bohusz \& Udalski 1979), the evidence was not yet compelling because of the remaining ambiguity in identifying the hump maxima and cycle counts, as already discussed by Patterson et al. (1981). Our continuous coverage of the entire outburst first unambiguously established the two periods of $0.056656(2) \mathrm{d}$ and $0.05726(1) \mathrm{d}$ for "early superhumps" and "genuine" superhumps, respectively ${ }^{2}$.

The main outburst terminated on August 16, but the rapid fading stopped on August 18 at 2 mag brighter than its quiescence level. After the dip for 5 days, twelve repetitive rebrightenings were observed between August 21 and September 13. This occurrence of rebrightenings far surpassed the past record of six rebrightenings in EG Cnc.

\section{Discussion}

The detection of the growth of early superhumps "after" the outbursts onset of AL Com and WZ Sge puts a period to the long-continuing argument between the competing two models; the mass-transfer burst model (Patterson et al. 1981) and the disk-instability model (Osaki 1995), originally continuing since 1970s. Although several different theoretical interpretations have been proposed as the cause of WZ Sge outbursts, no observational test has been available before the present outburst. We can now present the first firm observational evidence against the original mass-transfer burst model for the cause of WZ Sge outburst, proposed by Patterson et al. (1981). If the superoutburst is triggered by an enhanced mass-transfer, much larger humps due to the brightened hot spot are expected at the same time with the outburst onset. This is clearly

\footnotetext{
${ }^{2}$ The superhump period of $0.05714 \mathrm{~d}$ and the fractional superhump excess of $0.8 \%$ reported by Patterson et al. (1981) were found to be incorrect, which suffered from ambiguous identifications of superhumps.
} 
contrary to our observation. This is also supported by Doppler tomography of the earliest stage of the superoutburst (Baba et al. 2001b; Kuulkers et al. 2002). The strongest emission on velocity maps reside on the opposite side of the expected hot spot, excluding an increased mass-transfer as the origin of the strong emissions.

Some mechanisms for the early superhumps have been proposed by several authors. Our double-peaked profile (Fig. 4) excludes the hot spot model, since the profile would be single-peaked if the hot spot was the origin (Kato et al. 1996). Nogami et al. (1997) proposed a jet model and a thickened-edge disk model. Recently, a possibility, that the disk reaches the $3: 1$ resonance radius before the outburst, is pointed out (Meyer-Hofmeister et al. 1998), and we thought that the slightly distorted disk might be the origin. However, no consensus has been yet reached as to the origin of early superhumps.

Osaki \& Meyer (2001; in prep.) suggest that the double peaked light curve at the early stage of the outburst of WZ Sge stars is a manifestation of the 2:1 resonance at the accretion disk rim that was first investigate by Lin \& Papaloizou (1979). In binary systems with an extremely small mass ratio, the tidal force is very weak, and the accretion disk can extend so large that a strong 2:1 resonance begins to work (Lin \& Papaloizou 1979). A spiral dissipation pattern is produced by this resonance, which may draw a double-peaked light curve. The spiral pattern formed in the extended disk at the outburst onset may be a natural explanation of our observations.

We compared our photometric observations with the spectroscopic observations of WZ Sge. On July 23, just after the outburst onset, He II $\lambda 4686$ shows no emission componemt, but on July 24, the line was observed as a strong emission line. The Doppler maps of He II $\lambda 4686$ constructed using time-resolved spectra on July 24 and July 28 show that the accretion-disk emission is dominated by two spiral arms (Ishioka et al. 2001; Steeghs et al. 2001; Baba et al. 2001a; Baba et al. 2001c; Kuulkers et al. 2002). This result indicates that some relation exists between the growth of He II emission line and the spiral structure, and the observed growth of early superhumps. The doublepeaked profile of early superhumps may be explained by the two arms of the spiral structure ${ }^{3}$.

We propose that the physical reason, distinguishing the WZ Sge stars from other dwarf novae, is a very small mass ratio which allows the existence of the $2: 1$ resonance. The condition of the $2: 1$ resonance being inside the Rochelobe is the mass ratio of $q=M_{2} / M_{1} \leq 0.1$. The mass ratio is indicated from the superhump excess, $\epsilon$, which is expressed as $\epsilon=\left(P_{\mathrm{sh}}-P_{\text {orb }}\right) / P_{\text {orb }}$. WZ Sge stars clearly have small mass ratios compared with usual SU UMa stars.

\footnotetext{
${ }^{3}$ Spiral shocks are seen in other cataclysmic variables (e.g. IP Peg) that do not show similar humps. Also, since IP Peg has a long orbital period, neither the 2:1 nor the 3:1 resonances will occur in that star. However, the mechanisms driving spiral shocks of WZ Sge and above-the-gap dwarf novae can be different (Baba et al. 2001b).
}

Only several stars containing WZ Sge stars satisfy this criterion; the 2:1 resonance can be effective. We succeeded in understanding the WZ Sge stars in the unified way by a single mechanism.

Our observations with high time-resolution and long, continuous coverage not only revealed the behavior at the earliest stage of the outburst, rejecting the mass transfer instability model, but also lead the condition of $q \leq 0.1$, distinguishing WZ Sge stars from SU UMa stars. WZ Sge stars resemble X-ray transients containing black holes in some aspects. The investigation of WZ Sge stars is expected to contribute the understanding of the black hole transients with a similar mass ratio.

Acknowledgements. We are grateful to numerous collaborators of VSNET for the large contribution to this observation. We thank T. Ohshima for the discovery of the outburst at the earliest stage. We acknowledge Y. Osaki and D. Nogami for the fruitful discussion and useful advice. Part of this work was supported by a Research Fellowship of the Japan Society for the Promotion of Science for Young Scientists (M.U.).

\section{References}

Baba, H., Sadakane, K., \& Norimoto, Y. 2001a, IAU Circ., 7672

Baba, H., Sadakane, K., Norimoto, Y., et al. 2001b, PASJ, submitted

Baba, H., Sadakane, K., Norimoto, Y., et al. 2001c, IAU Circ., 7678

Bailey, J. 1979, MNRAS, 189, 41P

Bohusz, E., \& Udalski, A. 1979, IBVS, 1583

Henden, A. A., \& Landolt, A. U. 2001, IBVS, 5166

Ichikawa, S., Hirose, M., \& Osaki, Y. 1993, PASJ, 45, 243

Ishioka, R., Uemura, M., Matsumoto, K., et al. 2001, IAU Circ., 7669

Kato, T., Nogami, D., Baba, H., et al. 1996, PASJ, 48, L21

Kato, T., Sekine, Y., \& Hirata, R. 2001, PASJ, in press

Kuulkers, E., Knigge, C., Steeghs, D., Wheatley, P. J., \& Long, K. S. 2002, in The Physics of Cataclysmic Variables and Related Objects, ed. B. T. Gansicke, K. Beuermann, \& K. Reinsch (ASP), in press

Lin, D. N. C., \& Papaloizou, J. 1979, MNRAS, 186, 799

Matsumoto, K., Nogami, D., Kato, T., \& Baba, H. 1998, PASJ, 50,405

Meyer-Hofmeister, E., Meyer, F., \& Liu, B. F. 1998, A\&A, 339, 507

Nogami, D., Kato, T., Baba, H., et al. 1997, ApJ, 490, 840

Osaki, Y. 1989, PASJ, 41, 1005

Osaki, Y. 1995, PASJ, 47, 47

Patterson, J., Kemp, J., Skillman, D. R., et al. 1998, PASP, 110, 1290

Patterson, J., McGraw, J. T., Coleman, L., \& Africano, J. L. 1981, ApJ, 248, 1067

Skidmore, W. 1998, Ph.D. Thesis, Univ. Keele

Steeghs, D., Marsh, T. R., Kuulkers, E., \& Skidmore, W. 2001, IAU Circ., 7675

Warner, B. 1995, Cataclysmic Variable Stars (Cambridge University Press, Cambridge) 